\title{
Testing the Equivalence Principle in space with MICROSCOPE: the data analysis challenge
}

\author{
Joel Bergé $^{1}$, Quentin Baghi ${ }^{1}$ and Sandrine Pires ${ }^{2}$ \\ ${ }^{1}$ ONERA, the French Aerospace Lab, \\ 29 avenue de la Division Leclerc, 92320 Châtillon, France \\ email: joel.berge@onera.fr; quentin.baghi@onera.fr \\ ${ }^{2}$ Laboratoire AIM, CEA/DSM-CNRS, Université Paris Diderot, IRFU/SAp, CEA Saclay, \\ Orme des Merisiers, 91191 Gif-sur-Yvette, France \\ email: sandrine.pires@cea.fr
}

\begin{abstract}
Theories beyond the Standard Model and General Relativity predict a violation of the Weak Equivalence Principle (WEP) just below the current best experimental upper limits. MICROSCOPE (Micro-Satellite à traînée Compensée pour l'Observation du Principe d'Equivalence) will allow us to lower them by two orders of magnitude, and maybe to detect a WEP violation. However, analyzing the MICROSCOPE data will be challenging, mostly because of missing data and a colored noise burrying the signal of interest. In this communication, we apply an inpainting technique to simulated MICROSCOPE data and show that inpainting will help detect a WEP violation signal.
\end{abstract}

Keywords. Gravitation, Methods: data analysis, Methods: statistical

\section{Introduction}

Theories beyond the Standard Model and General Relativity (GR) are under development to solve the various challenges fundamental physics and cosmology are faced with. Among them, we can cite string theory and loop quantum theory, which aim to unify GR and quantum physics, or modified gravity theories such as scalar-tensor theories that aim to explain the accelerated expansion of the Universe with a modification of gravity on cosmological scales. Most of those theories predict a violation of the Weak Equivalence Principle (WEP).

The WEP states the equivalence of the gravitational mass $m_{g}$ and of the inertial mass $m_{i}$. It is a cornerstone of GR: any detection of a WEP violation will be a smoking gun for new physics, with significant implications for cosmology. Various experiments have been conducted over a century on the ground, bringing more and more stringent limits on possible WEP violations, by comparing how two bodies "A" and "B" made of different composition behave in the same gravitational field: the Eötvös ratio

$$
\eta=2 \frac{\left(m_{g} / m_{i}\right)_{A}-\left(m_{g} / m_{i}\right)_{B}}{\left(m_{g} / m_{i}\right)_{A}+\left(m_{g} / m_{i}\right)_{B}}
$$

quantifies a WEP violation. The best upper limit is currently $\eta \leqslant 10^{-13}$. As new gravity theories predict a WEP violation at the level $10^{-18} \leqslant \eta \leqslant 10^{-14}$, it is important to better test the WEP. Since on-ground experiments are now reaching their limits, it becomes important to bring experiments to space. MICROSCOPE is a French Space Agency funded mission which aims to test the WEP in space at the level $\eta=10^{-15}$, expected for launch in 2016 . 


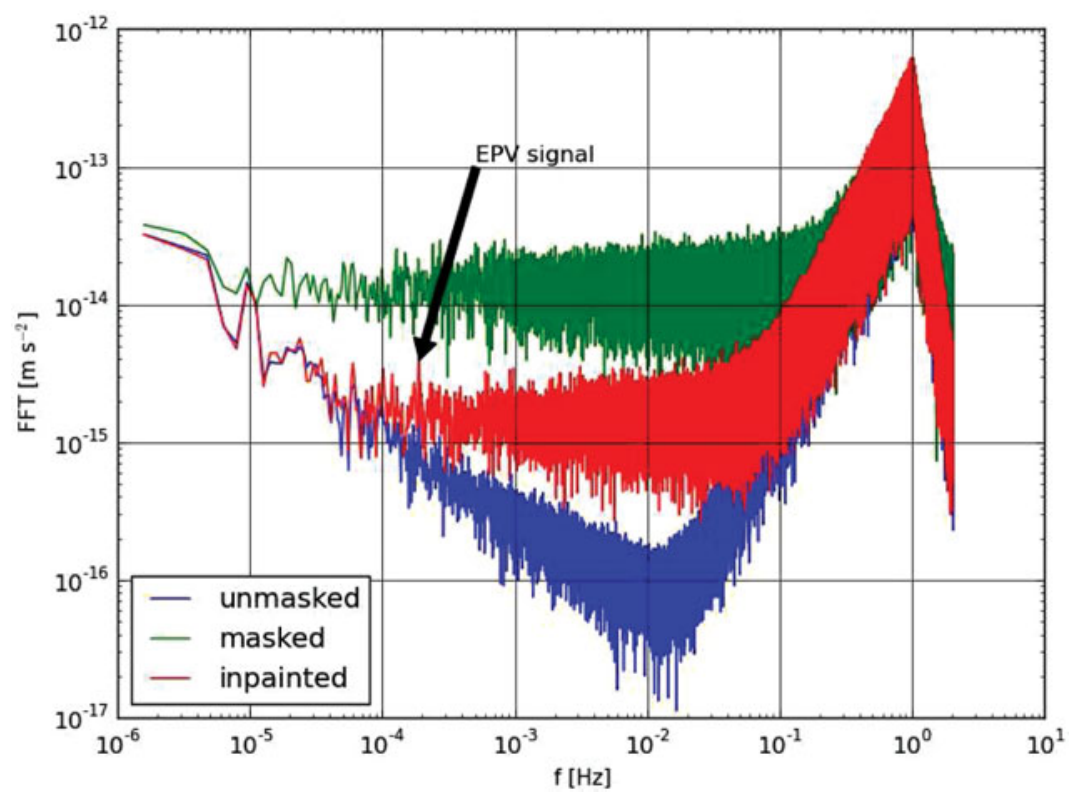

Figure 1. Linear power spectrum of simplified, simulated MICROSCOPE data. In blue, the original data; in green, the data with gaps; in red, the data with gaps filled in by inpainting. The simulation includes instrumental noise and a pure sine signal representing the WEP violation signal we are looking for, shown by the black arrow.

In this communication, we first present the MICROSCOPE mission, before emphasizing on its measurement challenge: detecting a very small signal burried in a colored noise and plagued by missing data.

\section{MICROSCOPE}

MICROSCOPE (Touboul 2009, Touboul et al. 2012) is a drag-free microsatellite which aims to test the WEP by comparing the acceleration experienced by two free-falling test masses in the Earth gravity field. It embarks two ultrasensitive electrostatic differential accelerometers. Each accelerometer is made up of two coaxial cylindrical proof masses whose motion is electrostatically constrained. In one (reference) accelerometer, the proof masses are made of the same material to demonstrate the experiment accuracy; they are made of different materials in the second accelerometer, which is used to test the WEP. The electric potential applied to keep the masses in equilibrium is a measure of the difference in the proof masses motion; hence, a non-zero applied potential is a measure of a WEP violation.

\section{Data analysis}

The MICROSCOPE data will consist of time series from which we will have to extract the signal of interest at a well known frequency that depends on the satellite's orbit and attitude. However, this signal will be burried in a colored stochastic noise, contaminated by various deterministic signals, and plagued by holes due to missing or invalid data. Therefore, its precise extraction and estimation require sophisticated methods. The blue curve in Fig. 1 shows the linear power spectrum of a simplified expected simulated signal: the instrumental noise increases both at low and high frequency, where it largely exceeds 
the amplitude of the expected WEP violation signal, shown by the "EPV signal" arrow. For the sake of simplicity, we did not simulate any contaminating deterministic signal.

Gaps in time series and invalid data are expected to occur because of several causes, such as telemetry loss, micrometeorite impacts, saturation due to mechanical crackles, and result in missing data (Hardy et al. 2013). They affect the measured power spectrum by a leakage of the high frequency noise into lower frequencies, where a WEP violation signal is looked for, as shown by the green curve in Fig. 1.

Here, we fill in gaps thanks to an inpainting technique, that has already been used to deal with missing data in several astrophysics applications (e.g. Pires et al. 2009, 2014): the missing information is extrapolated from the available data using a sparsity prior on the solution (Elad et al. 2005). Let $X(t)$ be the ideal complete time series, $Y(t)$ the measured incomplete time series and $M(t)$ the binary mask (1 where we have data, 0 elsewhere), such that $Y=M X$. Inpainting consists of recovering $X(t)$ knowing $Y(t)$ and $M(t)$. Among all the possible solutions, we search for the sparsest solution in a given representation $\phi^{T}$ (i.e. the time series $X$ that can be represented with the fewest coefficients $\left.\alpha=\phi^{T} X\right)$. The solution is obtained by solving min $\left\|\phi^{T} X\right\|_{0}$ under the constraint $\|Y-M X\|^{2} \leqslant \sigma^{2}$ where the $l_{0}$ norm is the number of non-zero coefficients, $\|z\|^{2}=\sum_{k} z_{k}^{2}$ is the classical $l_{2}$ norm and $\sigma$ is the noise rms. If the time series $X$ is sparse enough in the representation $\phi^{T}$ (i.e. few strong coefficients can represent the data), the $l_{0}$ pseudo-norm can be replaced by the convex $l_{1}$ norm, i.e. $\|z\|_{1}=\sum_{k}\left|z_{k}\right|$ (Donoho \& Huo 2001), and threfore its global minimum can be reached by descent techniques.

The red curve in Fig. 1 shows the result of using inpainting on our simulated MICROSCOPE data. The noise level (due to missing data) is clearly decreased, especially at low frequency, where we search for the WEP violation signal. From the figure, we see that after inpainting, it becomes possible to detect the peak due to the signal.

A weighted least square method can then be used to detect and characterize the WEP violation signal.

\section{Acknowledgements}

We thank Pierre Touboul, Gilles Métris, Emilie Hardy, Manuel Rodrigues and Bruno Christophe for useful discussions. This work makes use of technical data from the CNESESA-ONERA-CNRS-OCA Microscope mission, and has received financial support from ONERA and CNES. We acknowledge the financial support of the UnivEarthS Labex program at Sorbonne Paris Cité (ANR-10-LABX-0023 and ANR-11-IDEX-0005-02).

\section{References}

Donoho, D. \& Huo, X. 2001, IEEE Trans. Inf. Theory, 47, 2845

Elad, M., Starck, J.-L., Querre, P., \& Donoho, D. 2005, J. Appl. Comp. Harmon. Anal., 19, 340

Hardy, É., Levy, A., Métris, G., Rodrigues, M., \& Touboul, P. 2013, Space Sci. Rev., 180, 177

Pires, S., Starck, J.-L., Amara, A., et al. 2009, MNRAS, 395, 1265

Pires, S., et al. submitted

Touboul, P. 2009, Space Sci. Rev., 148, 455

Touboul, P., Métris, G., Lebat, V., \& Robert, A. 2012, Classical Quant. Grav., 29, 184010 